Short communication

\title{
Developing and testing a measure of COVID-19 organizational support of healthcare workers - results from Peru, Ecuador, and Bolivia
}

\author{
Stephen X. Zhang ${ }^{\mathrm{a}, *}$, Shuhua Sun ${ }^{\mathrm{b}}$, Asghar Afshar Jahanshahi ${ }^{\mathrm{c}}$, Aldo Alvarez-Risco ${ }^{\mathrm{d}}$, \\ Verónica García Ibarra ${ }^{\mathrm{e}}$, Jizhen Li ${ }^{\mathrm{f}}$, Ross Mary Patty-Tito ${ }^{\mathrm{g}}$ \\ ${ }^{a}$ Associate Professor, University of Adelaide, Adelaide, Australia \\ ${ }^{\mathrm{b}}$ Assistant Professor, Tulane University, New Orleans, USA \\ ${ }^{\mathrm{c}}$ Associate Professor, CENTRUM Católica Graduate Business School (CCGBS), Pontificia Universidad Católica del Perú (PUCP), Lima, Peru \\ ${ }^{\mathrm{d}}$ Associate Professor, Universidad de Lima, Lima, Peru

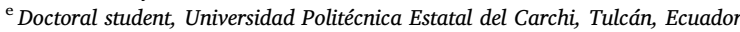 \\ ${ }^{\mathrm{f}}$ Associate Professor, Tsinghua University, Beijing, China \\ ${ }^{g}$ Caja Petrolera de Salud, La Paz, Bolivia
}

\section{A R T I C L E I N F O}

\section{Keywords:}

Pandemic

Anxiety

Life satisfaction

\begin{abstract}
A B S T R A C T
During the COVID-19 pandemic, healthcare workers are facing high workloads with resource constraints and risk of virus exposure, and healthcare organizations need to support their healthcare workers to reduce their anxiety. Based on a recent 8-point framework of COVID-19 specific organization support, we devised a measure of COVID-19 Organizational Support (COVID-OS). We tested the new measure with 712 healthcare workers in Bolivia, Ecuador, and Peru and found the new measure formed three factors to predict healthcare workers' anxiety and life satisfaction during the COVID-19 pandemic. We call for further studies to test COVID-OS in other countries and settings.
\end{abstract}

\section{Introduction}

Healthcare workers are playing crucial roles during the COVID-19 pandemic (Adalja et al., 2020). Yet, they also face great challenges such as the fear of being infected and infecting others, heavy workloads, and a lack of personal protection equipment (Liu et al., 2020; Shanafelt et al., 2020). A recent study showed a staggering $28 \%$ of healthcare workers surpassed the cut-off for anxiety disorders (Zhang et al., 2020a), impairing them from performing at their regular level (Shanafelt et al., 2020). To help alleviate the anxiety of the healthcare workers, healthcare organizations ought to provide specific support to address their workers' concerns during the pandemic. Yet, there is a lack of research on specific organizational support measures during this pandemic. This research is essential for evidence-based organizational support practices.

Through a literature review, we identified an 8-point framework of COVID-19 specific organizational support that Shanafelt et al. (2020) inducted from a series of interviews. Despite the intuitive appeal of the framework, the authors noted "few of these considerations and suggestions have substantial evidence to support them." This study used this framework to deduct an empirical measure of COVID-19
Organizational Support (COVID-OS) and empirically examined its associations with healthcare workers' anxiety and life satisfaction. Our measure and the empirical evidence help to enable research and practice on COVID-19 organizational support to alleviate healthcare staff anxiety.

\section{Methods}

Using an online survey via a region-stratified, two-stage cluster sampling, we reached 1203 healthcare workers in Bolivia, Ecuador, and Peru from April 10 to May 2, 2020. A total of 712 of them (response rate: $59.2 \%$ ) answered their demographic information, anxiety, life satisfaction, and the perceived COVID-19 Organizational Support (COVID-OS), deducted directly from the 8-point framework of Shanafelt et al. (2020) published in JAMA. Specifically, each organizational support item directly corresponds to a source of anxiety among healthcare workers based on the 8-point framework. These items ask the respondents to rate the extent to which they agree or disagree with each of eight statements using a 7-point Likert scale from 'strongly disagree' $(=1)$ to 'strongly agree' $(=7)$. The measure for COVID-OS can be found in Table 2 of Appendix A.

\footnotetext{
* Corresponding author.

E-mail address: stephen.x.zhang@gmail.com (S.X. Zhang).
} 


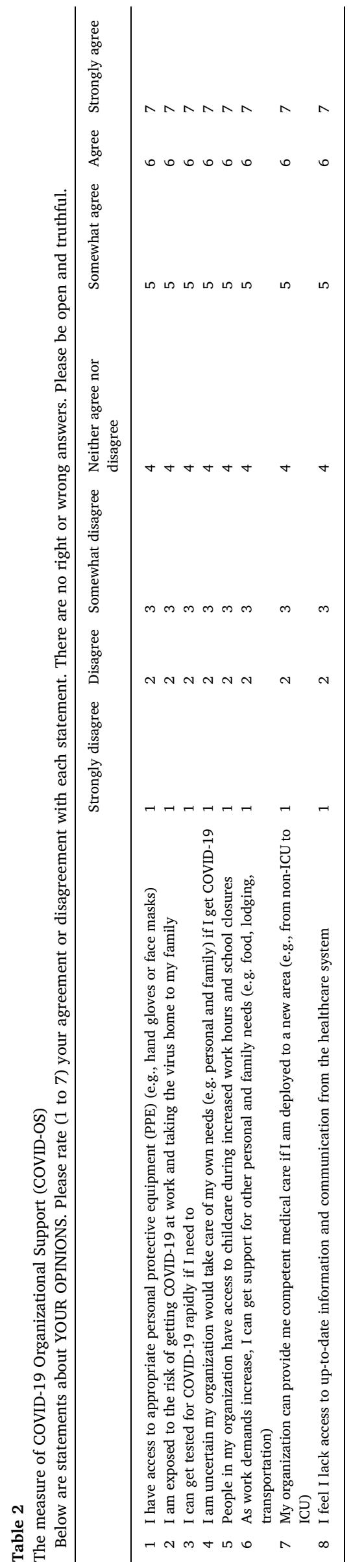

We assessed healthcare workers' anxiety using the 7-item GAD-7 scale. GAD-7 was developed to diagnose generalized anxiety disorder (Spitzer et al., 2006) with the following cut-offs: 0-4 (minimal symptoms, the reference group); 5-9 (mild symptoms), 10-14 (moderate symptoms), and 15-21 (severe symptoms). We measured healthcare workers' life satisfaction using the 5-item Satisfaction with Life Scale (SWLS-5). SWLS-5 was designed to measure global satisfaction with one's life (Diener et al., 1985). A score of 25-35 indicates life satisfaction; 5-14 indicates life dissatisfaction, and a score of 15-24 indicates neither strong life satisfaction or dissatisfaction.

Following scale development protocols (DeVellis, 2016), when there is a priori theory on the factor structure of the items, we used confirmatory factor analyses (CFA) to test this hypothesized factor structure (Hurley et al., 1997; Knekta et al., 2019). Nonetheless, we also compared a set of alternative models. After establishing the factor structure of the COVID-OS measure, we follow the procedure to test a psychiatric composite index by a set of analyses (Liu et al., 2015). First, we ran analyses treating GAD-7 and SWLS-5 as continuous variables to examine the predictive validity of the COVID-OS measure using OLS regression. Second, we used logistic regression to predict the clinical severity scores of GAD-7 and SWLS-5 using the cut-off values above to examine the clinical utility of the scale.

\section{Results}

Among the 712 healthcare workers, $68 \%$ were female, with average age of 38.9 years $(\mathrm{SD}=10.1)$. The majority of them were physicians (24\%), nurses (25\%), pharmacists (22\%), and administrators (8\%). Of the sample, 35\% were from Ecuador, 34\% from Bolivia and 31\% were from Peru. Using the cut-off points of varying degrees of anxiety, $44 \%$, $18 \%$ and $5 \%$ of the workers experienced mild, moderate, and severe anxiety disorders respectively. Using the cut-offs of the life satisfaction scale, $9 \%$ of the healthcare workers were not satisfied with their life and $58 \%$ found their life to be satisfactory. More descriptives can be found in the second column of Table 1.

Shanafelt et al. (2020) suggested that the eight points can be grouped into five themes. We thus used confirmatory factor analyses (CFA) to directly test their hypothesized 5-factor structure, but the CFA results demonstrated that the 5-factor model did not fit the data very well: $\quad\left(\chi^{2} \quad(13)=52.98, \quad p=0.000 ; \quad\right.$ CFI $=0.90 ; \quad$ TLI $=0.79$; RMSEA $=0.07)$. Given the items did not fit the predetermined structure, we reexamined the content and the data pattern to restructure the eight items into alternative dimensions. For instance, we tried to put the items into a single dimension and the CFA fit was poor $\left(\chi^{2}(20)=119.6\right.$; $\mathrm{CFI}=0.75$; TLI $=0.65$; RMSEA $=0.08$ ). A restructured 3 -factor model showed good CFA fit indices $\left(\chi^{2}(17)=38.22, p=0.002\right.$; $\mathrm{CFI}=0.95$; $\mathrm{TLI}=0.91$; RMSEA $=0.04$ ). Based on the item contents, we labeled the three factors as work support (items 1, 3, and 7), personal support (items 5 and 6), and risk support (items 2, 4, and 8). Further, the item intercorrelations suggest the items are better viewed as a formative measure (Willoughby et al., 2014).

We first ran OLS regression to examine the predictive validities of COVID-OS on anxiety and life satisfaction scores. Results revealed that work support $(b=-0.05 ; 95 \% \mathrm{CI}=[-0.08$ to -0.01$] ; p=0.012)$, personal support $(b=-0.04 ; 95 \% \mathrm{CI}=[-0.07$ to -0.01$] ; p=0.019)$ and risk support $(b=-0.05 ; 95 \% \mathrm{CI}=[-0.09$ to -0.00$] ; p=0.034)$ were all negatively associated with anxiety. Moreover, work support ( $b=0.22 ; 95 \% \mathrm{CI}=[0.14$ to 0.29$] ; p=0.000$ ) and personal support ( $b=0.15 ; 95 \% \mathrm{CI}=[0.09$ to 0.20$] ; p=0.000$ ) were also positively related to life satisfaction.

To check the clinical utility of the three factors of COVID-OS, they were used to predict the likelihood of anxiety disorders (mild; moderate; severe) and life satisfaction (dissatisfied; neither dissatisfied nor satisfied; satisfied). Ordered logistic regression analyses (Table 1) show personal support predicted less likelihood of mild anxiety. Work support predicted less likelihood of moderate anxiety. Both personal 
Table 1

Variable names, descriptive statistics, and the regression results on anxiety disorder (mild, moderate, and severe) and life satisfaction (satisfaction and dissatisfaction).

\begin{tabular}{|c|c|c|c|c|c|c|c|c|c|c|c|c|c|c|c|c|c|c|c|c|c|}
\hline \multirow{4}{*}{$\begin{array}{l}\text { Variable } \\
\\
\text { Female }\end{array}$} & \multirow{4}{*}{$\begin{array}{c}\text { Descriptives } \\
\begin{array}{c}\text { \% or mean } \\
\text { (st.d.) }\end{array} \\
68 \%\end{array}$} & \multicolumn{12}{|c|}{ DV: Anxiety } & \multicolumn{8}{|c|}{ DV: life satisfaction } \\
\hline & & \multicolumn{4}{|c|}{ Mild anxiety disorder } & \multicolumn{4}{|c|}{ Moderate anxiety disorder } & \multicolumn{4}{|c|}{ Severe anxiety disorder } & \multicolumn{4}{|c|}{ Life satisfaction } & \multicolumn{4}{|c|}{ Life dissatisfaction } \\
\hline & & \multirow{2}{*}{$\begin{array}{c}\text { O.R. } \\
1.45\end{array}$} & \multicolumn{2}{|c|}{$(95 \% C I)$} & \multirow{2}{*}{$\begin{array}{c}\boldsymbol{P} \\
0.052\end{array}$} & \multirow{2}{*}{$\begin{array}{c}\text { O.R. } \\
1.14\end{array}$} & \multicolumn{2}{|c|}{$(95 \% C I)$} & \multirow{2}{*}{$\begin{array}{c}\boldsymbol{P} \\
0.548\end{array}$} & \multirow{2}{*}{$\begin{array}{c}\text { O.R. } \\
0.76\end{array}$} & \multicolumn{2}{|c|}{$(95 \% C I)$} & \multirow{2}{*}{$\begin{array}{c}\boldsymbol{P} \\
0.452\end{array}$} & \multirow{2}{*}{$\begin{array}{c}\boldsymbol{O} . \boldsymbol{R} \text {. } \\
0.92\end{array}$} & \multicolumn{2}{|c|}{$(95 \% C I)$} & \multirow{2}{*}{$\begin{array}{c}\boldsymbol{P} \\
0.656\end{array}$} & \multirow{2}{*}{$\begin{array}{c}\text { O.R. } \\
0.94\end{array}$} & \multicolumn{2}{|c|}{$(95 \% \mathrm{CI})$} & \multirow{2}{*}{$\begin{array}{c}\boldsymbol{P} \\
0.841\end{array}$} \\
\hline & & & 1.00 & 2.10 & & & 0.74 & 1.74 & & & 0.36 & 1.58 & & & 0.63 & 1.34 & & & 0.50 & 1.76 & \\
\hline Age & $38.9(10.1)$ & 0.97 & 0.95 & 0.99 & 0.012 & 0.99 & 0.97 & 1.02 & 0.491 & 0.94 & 0.88 & 1.00 & 0.044 & 1.00 & 0.98 & 1.02 & 0.904 & 1.00 & 0.96 & 1.04 & 0.960 \\
\hline Children & $1.2(1.2)$ & 1.04 & 0.89 & 1.22 & 0.587 & 0.94 & 0.78 & 1.12 & 0.468 & 1.00 & 0.68 & 1.51 & 0.952 & 1.10 & 0.94 & 1.29 & 0.250 & 1.28 & 0.97 & 1.69 & 0.075 \\
\hline Education & $4.4(0.9)$ & 1.01 & 0.82 & 1.25 & 0.911 & 0.94 & 0.73 & 1.20 & 0.611 & 0.89 & 0.53 & 1.48 & 0.650 & 1.19 & 0.95 & 1.48 & 0.133 & 0.58 & 0.38 & 0.88 & 0.010 \\
\hline \multicolumn{22}{|l|}{ Job function } \\
\hline physician & $24 \%$ & & & & & & & $\mathbf{P}$ & 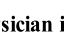 & th & . & e cat & & & & & & & & & \\
\hline nurse & $25 \%$ & 1.51 & 0.89 & 2.57 & 0.130 & 1.47 & 0.82 & 2.65 & 0.196 & 1.09 & 0.44 & 3.15 & 0.742 & 1.04 & 0.61 & 1.77 & 0.885 & 0.85 & 0.33 & 2.19 & 0.737 \\
\hline pharmacist & $22 \%$ & 1.03 & 0.62 & 1.73 & 0.902 & 1.36 & 0.73 & 2.56 & 0.335 & 0.67 & 0.20 & 2.48 & 0.592 & 1.34 & 0.80 & 2.27 & 0.270 & 1.03 & 0.39 & 2.70 & 0.955 \\
\hline technician/technologist & $11 \%$ & 1.82 & 0.87 & 3.78 & 0.110 & 2.01 & 0.91 & 4.44 & 0.085 & 0.34 & 0.06 & 2.47 & 0.317 & 1.25 & 0.62 & 2.54 & 0.534 & 0.40 & 0.08 & 1.89 & 0.246 \\
\hline administrator & $8 \%$ & 0.92 & 0.46 & 1.84 & 0.819 & 0.98 & 0.43 & 2.25 & 0.971 & 2.00 & 0.60 & 7.18 & 0.247 & 0.65 & 0.33 & 1.32 & 0.234 & 1.14 & 0.36 & 3.66 & 0.820 \\
\hline intern/student & $4 \%$ & 1.51 & 0.52 & 4.34 & 0.448 & 3.10 & 1.19 & 8.06 & 0.020 & 1.59 & 0.46 & 6.30 & 0.432 & 0.23 & 0.09 & 0.62 & 0.003 & 8.64 & 2.45 & 30.50 & 0.001 \\
\hline laboratorist/biochemist/biologist & $2 \%$ & 0.55 & 0.15 & 1.97 & 0.355 & 2.12 & 0.50 & 9.11 & 0.311 & 0.00 & 0.00 & . & 0.988 & 1.38 & 0.35 & 5.43 & 0.641 & 2.13 & 0.22 & 20.27 & 0.509 \\
\hline logistic/IT & $1 \%$ & 1.13 & 0.22 & 5.73 & 0.878 & 0.67 & 0.07 & 6.33 & 0.730 & 0.00 & 0.00 & . & 0.993 & 0.25 & 0.04 & 1.63 & 0.149 & 2.00 & 0.22 & 18.33 & 0.539 \\
\hline dentist & $1 \%$ & 3.38 & 0.37 & 30.93 & 0.281 & 2.55 & 0.43 & 14.95 & 0.300 & 0.00 & 0.00 & . & 0.988 & 1.49 & 0.19 & 11.46 & 0.704 & 2.67 & 0.22 & 31.90 & 0.438 \\
\hline others & $3 \%$ & 0.32 & 0.11 & 0.93 & 0.036 & 0.71 & 0.18 & 2.81 & 0.624 & 0.00 & 0.00 & . & 0.986 & 1.31 & 0.42 & 4.12 & 0.643 & 0.68 & 0.07 & 6.54 & 0.738 \\
\hline \multicolumn{22}{|l|}{ Country } \\
\hline Peru & $31 \%$ & & & & & & & & i & f & nce & egor & & & & & & & & & \\
\hline Ecuador & $35 \%$ & 0.64 & 0.40 & 1.02 & 0.061 & 1.36 & 0.83 & 2.23 & 0.227 & 0.75 & 0.43 & 3.26 & 0.741 & 4.31 & 2.71 & 6.86 & 0.000 & 0.33 & 0.14 & 0.75 & 0.009 \\
\hline Bolivia & $34 \%$ & 0.69 & 0.44 & 1.07 & 0.094 & 1.09 & 0.65 & 1.82 & 0.743 & 0.77 & 0.41 & 3.61 & 0.717 & 1.51 & 0.99 & 2.32 & 0.058 & 0.51 & 0.25 & 1.06 & 0.072 \\
\hline COVID-OS: work support & 4.11 & 0.94 & 0.82 & 1.08 & 0.381 & 0.85 & 0.74 & 0.98 & 0.021 & 0.96 & 0.74 & 1.25 & 0.757 & 1.46 & 1.28 & 1.67 & 0.000 & 0.66 & 0.52 & 0.83 & 0.000 \\
\hline COVID-OS: personal support & 2.96 & 0.84 & 0.75 & 0.95 & 0.004 & 0.98 & 0.86 & 1.11 & 0.731 & 1.03 & 0.81 & 1.33 & 0.758 & 1.24 & 1.09 & 1.40 & 0.001 & 0.69 & 0.54 & 0.88 & 0.002 \\
\hline COVID-OS: risk support & 3.29 & 0.89 & 0.77 & 1.03 & 0.131 & 0.91 & 0.78 & 1.06 & 0.232 & 0.84 & 0.63 & 1.14 & 0.276 & 1.04 & 0.90 & 1.20 & 0.581 & 0.81 & 0.64 & 1.02 & 0.074 \\
\hline
\end{tabular}

support and work support were associated with lower likelihood of life dissatisfaction and higher likelihood of life satisfaction. Risk support failed to predict anxiety and life satisfaction across the cut-off levels.

Moreover, our analyses also suggest that older healthcare staff were less likely to experience mild and severe anxiety and, compared with physicians, staff in other roles experienced less likelihood of mild anxiety, and interns/students had higher likelihood of moderate anxiety. Healthcare staff with lower education were more likely to be dissatisfied with their lives under COVID-19. Compared with physicians, interns/students had higher likelihood of being dissatisfied and lower likelihood of being satisfied with their lives.

Next, to examine the clinical utility of the scale in predicting the clinical caseness, we conducted analyses on the ROC (receiver operating characteristic) curve on the predictive ability for anxiety (GAD-
$7>=10$ ) with 1000 bootstraps. The results showed that the three factors and the covariates together resulted in an AUC of 0.61 with a sensitivity of 0.66 and a specificity of 0.56 by the Lin criteria (Fig. 1a in Appendix B), and the ROC is significantly better than the ROC of the demographic covariates alone. Furthermore, we used Item Response Theory (IRT) to investigate the properties of the eight items of COVID19 Organizational Support (AIC $=19,741.79$ as shown in Fig. $1 \mathrm{~b}$ in Appendix B).

\section{Discussion}

These results suggest that the eight items of COVID-19 Organizational Support formed three factors to predict healthcare workers' anxiety and life satisfaction during the COVID-19 pandemic.

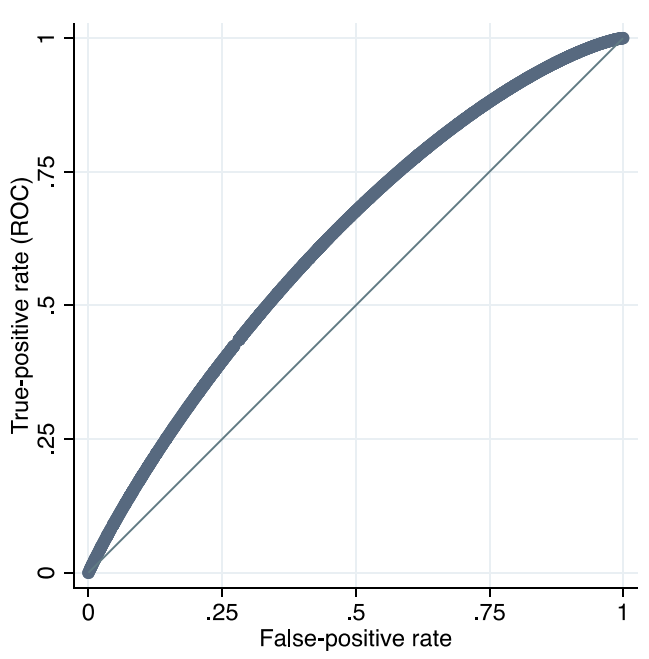

(a) ROC Curve

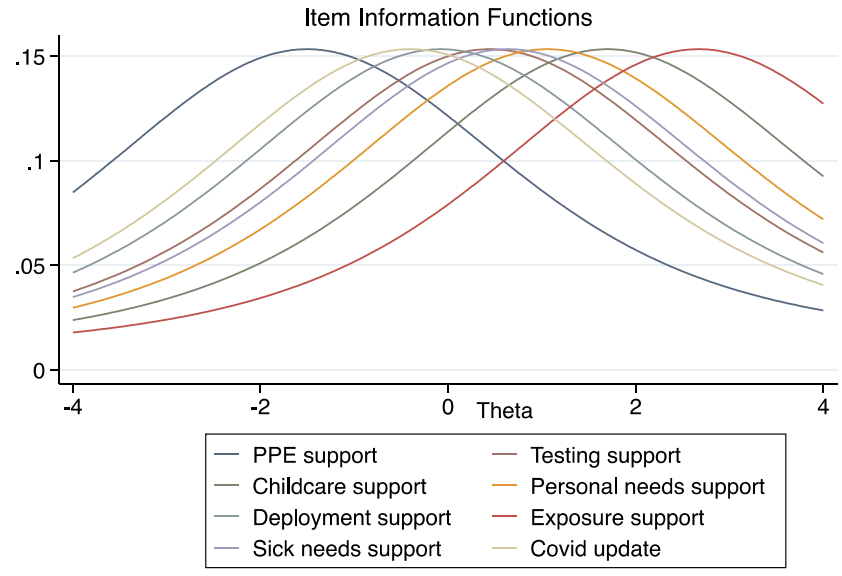

(b) Item information functions of the eight items of COVID-OS

Fig. 1. (a) The (ROC) receiver operator characteristic curve; (b) item information functions of the eight items of COVID-OS [COVID-19 Organizational Support]. 
Linear regression showed all three factors of organization support predicted lower anxiety, and work support and personal support also predicted higher life satisfaction. Ordered logistic regression showed personal support and work support each predicted different levels of anxiety, and levels of life (dis)satisfaction. Risk support did not play a significant role in our sample. Risk support may not be significant because healthcare workers had treated risks as an inherent part of their jobs during the COVID-19 pandemic. Together, these findings suggest that healthcare organizations should consider prioritizing personal and work support under resource constraints.

This study has limitations. First, as the study is limited to three countries with varying degrees of COVID-19 infection, the generalizability to other countries remains an empirical question for future research. Second, we focused on healthcare workers. However, as many countries are relaxing restrictions and opening up, other workers will return to work (Zhang et al., 2020b), and their organizations also similarly need to identify specific organization support to allow their employees to work without anxiety. Future research may consider applying and adapting our COVID-OS support measure in other contexts.

The measure of COVID-OS allows healthcare organizations to assess, monitor, compare and improve COVID-19 specific support to their healthcare workforce to mitigate their anxiety and fear while working during the pandemic. The measure can easily be implemented via an anonymous survey to help reveal important areas of concern that might be harder to uncover otherwise.

\section{Appendix}

Supplementary graphs on the property of the measure of COVID-19 Organizational Support (COVID-OS)

\section{CRediT authorship contribution statement}

Stephen X. Zhang: Conceptualization, Investigation, Methodology, Formal analysis, Visualization, Writing - original draft, Writing - review \& editing, Supervision. Shuhua Sun: Conceptualization, Investigation, Writing - original draft, Writing - review \& editing. Asghar Afshar Jahanshahi: Investigation, Resources, Conceptualization, Writing review \& editing. Aldo Alvarez-Risco: Investigation. Verónica García Ibarra: Investigation. Jizhen Li: Resources, Funding acquisition. Ross Mary Patty-Tito: Investigation.

\section{Declaration of Competing Interests}

The authors declare that there are no potential conflicts of interest with respect to the research, authorship, and/or publication of this article.

\section{Acknowledgment}

We acknowledge the support of Tsinghua University-INDITEX Sustainable Development Fund (Project No. TISD201904) and the assistance from Meghana Kotha, CA, USA.

\section{Supplementary materials}

Supplementary material associated with this article can be found, in the online version, at doi:10.1016/j.psychres.2020.113174.

\section{References}

Adalja, A.A., Toner, E., Inglesby, T.V., 2020. Priorities for the US health community responding to COVID-19. JAMA 323, 1343. https://doi.org/10.1001/jama.2020.3413.

DeVellis, R., 2016. Scale development: Theory and Applications, 26th ed. Sage, London.

Diener, E., Emmons, R.A., Larsen, R.J., Griffin, S., 1985. The satisfaction with life scale. J. Pers. Assess. 49, 71-75. https://doi.org/10.1207/s15327752jpa4901_13.

Hurley, A.E., Scandura, T.A., Schriesheim, C.A., Brannick, M.T., Seers, A., Vandenberg, R.J., Williams, L.J., 1997. Exploratory and confirmatory factor analysis: guidelines, issues, and alternatives. J. Organ. Behav. 18, 667-683 10.1002/(SICI)10991379(199711)18:6 <667::AID-JOB874 > 3.0.CO;2-T.

Knekta, E., Runyon, C., Eddy, S., 2019. One size doesn't fit all: using factor analysis to gather validity evidence when using surveys in your research. CBE-Life Sci. Educ. 18, rm1. https://doi.org/10.1187/cbe.18-04-0064.

Liu, H., Prause, N., Wyatt, G.E., Williams, J.K., Chin, D., Davis, T., Loeb, T., Marchand, E., Zhang, M., Myers, H.F., 2015. Development of a composite trauma exposure risk index. Psychol. Assess. 27, 965-974. https://doi.org/10.1037/pas0000069.

Liu, Q., Luo, D., Haase, J.E., Guo, Q., Wang, X.Q., Liu, S., Xia, L., Liu, Z., Yang, J., Yang, B.X., 2020. The experiences of health-care providers during the COVID-19 crisis in China: a qualitative study. Lancet Glob. Heal. 0. https://doi.org/10.1016/S2214109X(20)30204-7.

Shanafelt, T., Ripp, J., Trockel, M., 2020. Understanding and addressing sources of anxiety among health care professionals during the COVID-19 pandemic. JAMA. https://doi.org/10.1001/jama.2020.5893.

Spitzer, R.L., Kroenke, K., Williams, J.B.W., Löwe, B., 2006. A brief measure for assessing generalized anxiety disorder. Arch. Intern. Med. 166, 1092. https://doi.org/10.1001/ archinte.166.10.1092.

Willoughby, M., Holochwost, S.J., Blanton, Z.E., Blair, C.B., 2014. Executive functions: formative versus reflective measurement. Meas. Interdiscip. Res. Perspect. 12, 69-95. https://doi.org/10.1080/15366367.2014.929453.

Zhang, S.X., Liu, J., Jahanshahi, Afshar, Nawaser, A., Yousefi, K., Li, A., Sun, J., S., 2020a At the height of the storm: healthcare staff's health conditions and job satisfaction and their associated predictors during the epidemic peak of COVID-19. Brain. Behav. Immun. https://doi.org/10.1016/J.BBI.2020.05.010.

Zhang, S.X., Sun, S., Jahanshahi, A.A., Wang, Y., Madavani, A.N., Dinani, M.M., 2020b. Beyond predicting the number of infections: predicting who is likely to be COVID negative or positive. medRxiv 2020 04, 30. https://doi.org/10.1101/2020.04.30. 20086348. 20086348. 